\title{
Chios Mastic Gum Consumption Has a Protective Effect on Ovariectomy-Induced Bone Loss in Rats
}

\author{
Angeliki Eirini Pepe ${ }^{1}$, Pavlos Lelovas ${ }^{1}$, Vasiliki K. Pachi ${ }^{2}$, Maria Halabalaki ${ }^{2}$, Antonis Galanos ${ }^{1}$, \\ Panagiota Mikou, Dimitrios Pitidis Poutos ${ }^{4}$, George Papadomichelakis ${ }^{5}$, Stavros Kourkoulis ${ }^{6}$, \\ Ermioni Pasiou ${ }^{6}$, Sofia Mitakou $^{2}$, Theodore Karatzas ${ }^{7}$, Angeliki Triantafyllou ${ }^{1 *}$, and Ismene A. Dontas ${ }^{1 *}$ \\ ${ }^{1}$ Laboratory for Research of the Musculoskeletal System, School of Medicine, National \& Kapodistrian University of Athens, \\ KAT Hospital, Kifissia 14561, Greece \\ ${ }^{2}$ Division of Pharmacognosy \& Natural Products Chemistry, Department of Pharmacy, National and Kapodistrian University of Athens, \\ Zographou 15771, Greece \\ ${ }^{3}$ Department of Cytology, Laiko Hospital, Athens 11527, Greece \\ ${ }^{4}$ Department of Neurology, Evangelismos Athens General Hospital, Athens 10676, Greece \\ ${ }^{5}$ Department of Nutritional Physiology and Feeding, Faculty of Animal Science and Aquaculture, Agricultural University of Athens, \\ Athens 11855, Greece \\ ${ }^{6}$ Laboratory of Testing and Materials, Unit of Biomechanics, Department of Mechanics, National Technical University of Athens, \\ Zographou 15780, Greece \\ ${ }^{7}$ 2nd Propedeutic Department of Surgery / Laboratory of Experimental Surgery \& Surgical Research 'N.S. Christeas' School of Medicine, \\ National and Kapodistrian University of Athens, Athens 11527, Greece
}

\begin{abstract}
The purpose of this study was to examine the potential effect of Chios Mastic Gum (CMG) consumption on bone mineral density (BMD) and strength of ovariectomized rats. CMG is a known resin used from ancient times for its beneficial biological properties. Thirty mature female Wistar rats were randomized into three equal groups: sham-operated (control), ovariectomized (OVX), and ovariectomized and administered CMG per os (OVX+CMG). BMD of the total tibia, proximal tibia, and the 6th lumbar vertebra were measured at baseline and at 3 and 6 months post ovariectomy. Bone strength was assessed with three-point-bending $(3 \mathrm{pb})$ of the right femur. At 3 and 6 months, BMD values of the OVX+ CMG group were significantly higher for the anatomical cites evaluated than those of the OVX group. Femoral thickness assessed via $3 \mathrm{pb}$ had intermediate values in the treated group compared to the other groups. Cytology of vaginal smears and uterine weight of the $\mathrm{OVX}+\mathrm{CMG}$ group were consistent with estrogen depletion. Gastrocnemius muscle and intraperitoneal fat ratios to body weight (BW) of the OVX+CMG group did not significantly differ from the control group. Daily consumption CMG had a protective effect on BMD of the total and proximal tibia and the 6th lumbar vertebra of the rats, without causing undesirable effects on the vaginal epithelium and uterus. The $3 \mathrm{pb}$ results also demonstrated a favorable effect on the thickness of rat femurs. In addition, CMG was beneficial for both the muscular system and the intraperitoneal fat/BW ratio of the rats.
\end{abstract}

Keywords: bone mineral density, Chios Mastic Gum, cytological evaluation, osteoporosis, rat

\section{INTRODUCTION}

Osteoporosis is a metabolic bone disease characterized by microarchitectural degeneration of bone tissue and low bone mass. Reduced bone strength and increased bone fragility are caused by an imbalance in bone metabolism, whereby the ratio of bone resorption to bone formation is increased (Rachner et al., 2011). In osteoporosis, lipid peroxidation is increased due to reductions in antioxidants (Maggio et al., 2003), since reactive oxygen species play a role in bone metabolism (Sontakke and Tare, 2002). Furthermore, inflammation modulates bone resorption via two mechanisms: tumor necrosis factor-related activation-induced cytokines and macrophage col- 
ony stimulating factors (Lacativa and de Farias, 2010). Loss of estrogen accelerates the effects of aging on bone by decreasing defense against oxidative stress, which leads to bone loss (Almeida et al., 2007).

Currently, a variety of pharmacological approaches are used in the treatment of postmenopausal osteoporosis. Most of these therapies have a variety of side effects (Rossouw et al., 2002; Beral et al., 2007). Therefore, there is an increased interest in alternative therapies for treatment of postmenopausal osteoporosis. Several plant extracts are being investigated for their beneficial effect on bone health, which may be attributed to their phyto-estrogenic and/or antioxidant action.

Chios Mastic Gum (CMG) is produced from an evergreen bush of the Anacardiaceae family Pistacia lentiscus var. Chia cv. Anacardiaceae. Information about its traditional use can be found in ancient texts of Galen, Theophrastus, and Dioscorides (Pachi et al., 2020). In 2015, CMG was officially recognized as a traditional herbal medicinal product by the European Medicines Agency (2015). From chemical point of view, $65 \sim 70 \%$ of all constituents of CMG are terpenes, which makes it unique amongst other natural resins. The most characteristic terpenes of CMG belong to triterpenoids (Giaginis and Theocharis, 2011), which have a significant protective effect on bone mineral density in ovariectomized mice ( $\mathrm{Li}$ et al., 2007).

In addition, Cimicifugae rhizome (black cohosh), a well investigated and officially accepted herbal medicine for menopause complications, is characterized by the presence of terpenoids (European Medicines Agency, 2017), and has protective effects against osteoporosis ( $\mathrm{Li}$ and $\mathrm{Yu}$, 2006). However, C. rhizome mainly contains cycloartanetype terpenoids whereas CMG from oleanane, tirucallenne, lupine, lupan, and dammarane derivatives have been reported to contain at least 120 different terpenoids. To the best of our knowledge, the effect of CMG has not been yet examined in mature ovariectomized rats, which is a well-documented model for the study of postmenopausal osteoporosis (Jee and Yao, 2001).

Mature ovariectomized rats are considered a valuable model due to similarities between the skeletons of rats and humans in terms of pathophysiological and due to husbandry and economic advantages (Lelovas et al., 2008). Given that CMG has anti-inflammatory properties (Triantafyllou et al., 2011) and inflammation modulates bone resorption (Lacativa and de Farias, 2010), the present study aimed to examine if CMG may have a beneficial effect on bone in the mature ovariectomized rat model of osteoporosis. Since CMG is a complex natural resin containing at least 120 chemical compounds, CMG was used in its entirety (Pachi et al., 2020).

\section{MATERIALS AND METHODS}

\section{Experimental animals}

The experimental protocol was approved by the General Directorate of Veterinary Services according to Greek legislation (license number 2158/18.06.2015; Presidential Decree 56/2013, in compliance with the European Directive 2010/63/EU) (Protocol Evaluation Committee, https://eur-lex.europa.eu/LexUriServ/LexUriServ.do?uri $=$ OJ:L:2010:276:0033:0079:en:PDF).

Thirty mature virgin female Wistar rats aged 10 months were included in this study. The appropriate selection of age is of vital importance since bone changes observed in rat skeletons after 9 months of age closely resemble those of the adult human skeleton (Erben, 1996; Jee and Yao, 2001; Lelovas et al., 2008). Rats were housed three per cage in transparent polycarbonate cages $(45 \times 3 \times 20 \mathrm{~cm}$; IFFA) and maintained under standard laboratory conditions (temperature, 19 to $22^{\circ} \mathrm{C}$; relative humidity, 55 to 65\%; 15 air changes/h; light/dark cycle at 06:00/18:00 h). The animals were randomly divided into three groups: sham-operated (control, $n=10$ ); ovariectomized without treatment (OVX, $\mathrm{n}=10)$; and ovariectomized and administered CMG (OVX+CMG, $\mathrm{n}=10)$ per os, immediately post-operatively.

We calculated that a sample size of 10 rats per group was required for an $80 \%$ probability of demonstrating a difference between groups of $>10 \%$ [standard deviation $(\mathrm{SD})=5$ ] in percentage change from baseline to the sixth month of bone mineral density (BMD) (OVX: $-35 \pm 6 \%$, OVX+CMG: $-25 \pm 6 \%$ ) with a significance of $<1.7 \%$ (two tailed test with Bonferroni correction). The sample size estimation was performed using G*Power 3.1.9.2 program (Heinrich-Heine-Universität Düsseldorf, Düsseldorf, Germany).

All rats were fed the Mucedola 4RF21 diet (Mucedola, Milan, Italy), which did not contain soy or soy by-products to avoid any estrogenic effects on BMD. Rat chow was given to the OVX+CMG group was enriched with CMG. Briefly, the chow was ground using a hammer mill through a $5 \mathrm{~mm}$ screen to obtain a mash. The mash was then mixed with powdered CMG in a rotary mixer to achieve a $14.35 \mathrm{mg} \mathrm{CMG} / \mathrm{kg}$ diet, and was pelleted using a pellet press (model 3872FPP24, R A Lister and Company Ltd., Dursley, UK). The total amount of food was equal to that given to the control group, in order to avoid obesity of the ovariectomized rats. The daily mean consumption of food was $20 \mathrm{~g} / \mathrm{d} / \mathrm{rat}$ and all rats had free access to drinking water. Food and water consumption were monitored three times per week, and body weight (BW) was measured once per two weeks. All BMD measurements were performed in vivo. The right femurs were harvested after euthanasia and properly stored according to Turner and Burr (Turner and Burr, 1993) for three- 
point-bending $(3 \mathrm{pb})$ bone biomechanical testing, which was performed one month after euthanasia.

\section{CMG}

CMG powder was kindly provided by the Chios Mastiha Growers Association. The dose of CMG administered to the rats was $0.83 \mathrm{~g} / \mathrm{kg}$ of $\mathrm{BW} / \mathrm{d}$, which is equivalent to 8 $\mathrm{g} / \mathrm{d}$ for a $60 \mathrm{~kg}$ human or $133 \mathrm{mg} / \mathrm{kg}$ of BW. For the conversion of the human dose to the equivalent rat dose we used the formula: human equivalent dose (HED, $\mathrm{mg} / \mathrm{kg})=$ animal dose $(\mathrm{mg} / \mathrm{kg}) \times($ animal $\mathrm{Km} / \mathrm{human}$ $\mathrm{Km}$ ) (Nair and Jacob, 2016), where HED is the human equivalent dose, animal $\mathrm{Km}$ is a factor that depends on the laboratory animal species (6 for rats) and human $\mathrm{Km}$ factor is 37 for a $60 \mathrm{~kg}$ human.

\section{Chemical analysis of CMG}

CMG was analyzed using an ACQUITY UPLC system (Waters Corporation, Milford, MA, USA) hyphenated to an LTQ-Orbitrap XL hybrid mass spectrometer (Thermo Fisher Scientific, Waltham, MA, USA), and electrospray ionization (ESI) in negative mode. The solvent system consisted of (A) $0.1 \%$ formic acid and (B) acetonitrile, and the flow rate was set at $0.4 \mathrm{~mL} / \mathrm{min}$. For separation, a Supelco Ascentis Express C18 $(100 \times 2.1 \mathrm{~mm}$ i.d., 2.7 $\mu \mathrm{m}$ particle size, Sigma-Aldrich Co., St. Louis, MO, USA) column was used, with the following elution gradient: 5 70\% B for $3 \mathrm{~min}$; 70 100\% B for $17 \mathrm{~min}$; stop for 4 min; $5 \sim 100 \%$ B for $1 \mathrm{~min}$; $3 \mathrm{~min}$ equilibration time. The injection volume was set to $10 \mu \mathrm{L}$.

A sample-preparation step was followed prior the analysis of CMG. This step is required in order to remove polymers (poly- $\beta$-myrcene) disturbing the analysis, allowing the detection of triterpenes. Specifically, CMG was dissolved in ethyl acetate and methanol was added following the procedure of Paraschos and colleagues (2007). After a resting period of 2 days, the polymer layer was decanted. The supernatant solution was filtrated (polyvinylidene fluoride, $0.45 \mu \mathrm{m}$ ) and evaporated to dryness. Water : acetonitrile (1:1) was used for sample dilution at a concentration of $100 \mu \mathrm{g} / \mathrm{mL}$. All data from full scan high resolution mass spectrometry (HRMS) \& HRMS/ mass spectrometry (MS) was acquired over a mass range of $\mathrm{m} / \mathrm{z} 115 \sim 1,000$.

To identify the extract constituents, chromatographic and spectrometric characteristics were used alongside published specifically retention time, proposed elemental composition (EC), ring double bond equivalent values (RDBeq.), HRMS/MS spectra and fragmentation patterns (Lemonakis et al., 2011; Andreadou et al., 2016; Xynos et al., 2018), and natural products databases (PubChem and ChemSpider). The raw data were obtained and processed using XCalibur software version 2.2TM (Thermo Fisher Scientific).

\section{Bone mineral density measurements}

The BMD of all rats was measured with dual-energy X-ray absorptiometry (DEXA) at baseline and at 3 and 6 months after ovariectomy in vivo. Before BMD measurements, rats were anaesthetized with intramuscular injections of $0.25 \mathrm{mg} / \mathrm{kg}$ dexmedetomidine and $50 \mathrm{mg} / \mathrm{kg}$ ketamine. For BMD measurements, a GE Lunar Prodigy densitometer machine (GE Healthcare, Chicago, IL, USA) with small animal software was used.

During the analysis, the evaluator was blinded and three different regions of interest (ROI) were positioned. The first ROI contained the whole left tibia, which is comprised of cortical and trabecular bone. The second ROI was a square of $0.19 \times 0.19 \mathrm{~mm}^{2}$, placed in the proximal tibia metaphysis, $1 \mathrm{~mm}$ from the epiphyseal line. The third ROI was a square of $0.32 \times 0.32 \mathrm{~mm}^{2}$ placed in the 6 th lumbar vertebra. The system was calibrated before every measurement.

\section{Ovariectomy}

After the first BMD measurement, ovariectomy was performed to all rats except the control group. Before the operation, rats were anaesthetized by dexmedetomidine and ketamine, as previously described. Analgesia and chemoprophylaxis were administered by subcutaneous injection of $4 \mathrm{mg} / \mathrm{kg}$ of carprofen and $10 \mathrm{mg} / \mathrm{kg}$ of enrofloxacin, respectively. Bilateral ovariectomy was performed under aseptic conditions.

\section{Cytologic evaluation of vaginal smears}

The impact of CMG on the vaginal epithelial maturation was assessed as a criterion of estrogenic effects. Vaginal smears were examined cytologically for five consecutive days before euthanasia by assessing cornification indexes as indicators of estrogenic effects (Goldman et al., 2007).

\section{Euthanasia and specimen collection}

After 6 months, rats were anaesthetized by dexmedetomidine and ketamine, as previously described, and were euthanized by an intravenous overdose of sodium pentobarbital through the caudal vena cava. To verify the success of ovariectomy and examine for malignancies or different pathologic findings, a complete autopsy was performed. The success of the ovariectomy was verified by the absence of ovarian tissue and by atrophy of the uterus. The uterus, liver, left kidney, intraperitoneal fat, and gastrocnemius muscle were carefully removed and weighed immediately after euthanasia on a digital microscale. Right femurs were excised, cleaned of adhering soft tissues, and stored as described below.

\section{Biomechanical testing}

Bending tests are well-documented and valid procedures for evaluating biomechanical properties of bones in small 
animals, such as rodents (Turner and Burr, 1993). In our study, the mechanical strength of right femurs were assessed ex vivo by the $3 \mathrm{pb}$ method. The right femur of each rat was collected during euthanasia and stored at $-20^{\circ} \mathrm{C}$ wrapped in gauzes immersed in normal saline (Turner and Burr, 1993).

\section{Statistical analysis}

Data were expressed as mean \pm SD, and Shapiro-Wilks tests were used for normality analysis of the parameters. Comparisons of BMD parameters at each time point, 3pb results, BW, organ weights, and ratios of organ weight/ $\mathrm{BW}$ between groups were performed using the one-way analysis of variance (ANOVA), and pairwise comparisons were performed using Bonferroni tests. One factor repeated measures ANOVA tests were used to compare different time measurements of BMD parameters for each group. Pairwise multiple comparisons were performed using Bonferroni tests.

Mean percentage changes from baseline after 3 and 6 months were calculated for BMD measurements. Comparisons between groups of percentage changes in BMD parameters from baseline during the observation period were analyzed using the one-way ANOVAs, and pairwise comparisons were performed using Bonferroni tests. Kruskal Wallis and Mann-Whitney tests were used in cases of violation of normality.

All tests were two-sided and statistical significance was set at $P<0.05$. All analyses were performed using the statistical package SPSS ver. 21.00 (IBM Corporation, Armonk, NY, USA).

\section{RESULTS}

\section{Chemical analysis}

In the current study, CMG was qualitatively characterized using ultra performance liquid chromatography-HRMS, as described by Andreadou and coworkers (Andreadou et al., 2016). The LC-HRMS base peak chromatogram of CMG used in this study is presented in Fig. 1, demonstrating the presence of masticadienonic acid (MNA), isomasticadienonic acid (IMNA), and other triterpenic acids, such as oleanonic, moronic, and olean-12,18-dien3-olic acid.

\section{BMD absolute values}

Total tibia: The results of the total tibia measurements are presented in Table 1. At baseline, there were no significant differences between BMD values between treatment groups. The BMD of the control group increased during the first 3 months $(P=0.024)$ and then stabilized during the next 3 months. However, the BMD significantly decreased from baseline at 3 and 6 months in both the OVX and OVX+CMG groups (both $P<0.001$ ). The BMD was significantly higher in the OVX+CMG group than the OVX group at both 3 and 6 months (both $P<0.001$ ).

Proximal tibia: The results of the proximal tibia measurements are presented in Table 2. In the control group, BMD of the proximal tibia did not significantly change during the observation period. However, in the OVX and OVX + CMG groups, the BMD significantly decreased after 3 (both $P<0.001$ vs. baseline) and 6 months (both $P<$ 0.001 vs. 3 months). The BMD of the OVX+CMG group

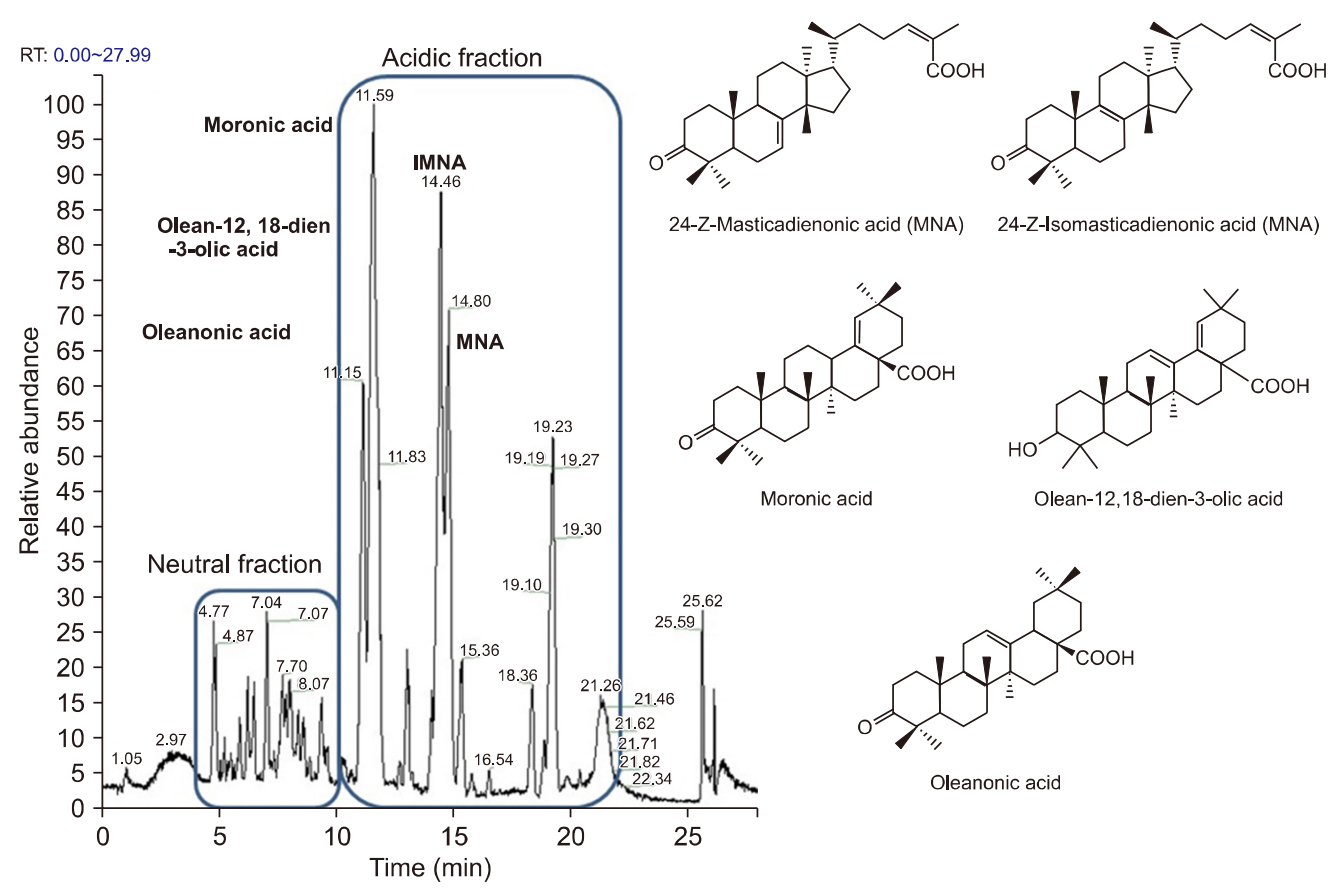

Fig. 1. UPLC-ESI(-)-HRMS full scan chromatogram of the Chios Mastic Gum used in the study. The peaks and the structures of the major triterpenic acids are annotated. 
Table 1. Comparison of the total tibia between groups during the observation period of 6 months

\begin{tabular}{lcccccc}
\hline \multicolumn{1}{c}{ Group } & Baseline & 3 Months & 6 Months & $\begin{array}{c}P \text { value } \\
\text { within } \\
\text { group }\end{array}$ & $\begin{array}{c}\% \text { Change } \\
\text { baseline to } \\
3 \text { months }\end{array}$ & $\begin{array}{c}\% \text { Change } \\
\text { baseline to } \\
6 \text { months }\end{array}$ \\
\hline Control & $0.243 \pm 0.012$ & $0.254 \pm 0.009^{\#}$ & $0.255 \pm 0.013$ & 0.047 & $4.41 \pm 4.15$ & $5.01 \pm 8.03$ \\
OVX & $0.248 \pm 0.010$ & $0.217 \pm 0.015^{\dagger *}$ & $0.205 \pm 0.007^{\dagger *}$ & $<0.001$ & $-12.71 \pm 5.08^{\dagger}$ & $-17.46 \pm 3.85^{\dagger}$ \\
OVX + CMG & $0.252 \pm 0.018$ & $0.231 \pm 0.009^{\dagger *}$ & $0.224 \pm 0.007^{\dagger *}$ & $<0.001$ & $-7.95 \pm 6.82^{\dagger}$ & $-10.63 \pm 6.75^{\dagger}$ \\
$P$ value between groups & 0.390 & $<0.001$ & $<0.001$ & $<0.0005$ & $<0.0005$ \\
\hline
\end{tabular}

Comparison of absolutes values $\left(\mathrm{g} / \mathrm{cm}^{2}\right)$ and mean percentage changes of bone mineral density of the total tibia among groups measured at baseline (pre-ovariectomy), and at 3 and 6 months post-ovariectomy.

Values are mean \pm SD.

Significant differences at ${ }^{*} P<0.001$ and ${ }^{\#} P=0.024$ vs. baseline, ${ }^{\dagger} P<0.001$ vs. control, and ${ }^{\ddagger} P<0.001$ vs. OVX.

OVX, ovariectomy; OVX + CMG, ovariectomy + Chios Mastic Gum.

Table 2. Comparison of the proximal tibia between groups during the observation period of 6 months

\begin{tabular}{lcccccc}
\hline \multicolumn{1}{c}{ Group } & Baseline & 3 Months & 6 Months & $\begin{array}{c}P \text { value } \\
\text { within } \\
\text { group }\end{array}$ & $\begin{array}{c}\% \text { Change } \\
\text { baseline to } \\
3 \text { months }\end{array}$ & $\begin{array}{c}\% \text { Change } \\
\text { baseline to } \\
6 \text { months }\end{array}$ \\
\hline Control & $0.413 \pm 0.022$ & $0.421 \pm 0.021$ & $0.425 \pm 0.027$ & 0.328 & $2.05 \pm 5.98$ & $2.89 \pm 5.56$ \\
OVX & $0.405 \pm 0.024$ & $0.290 \pm 0.026^{\dagger *}$ & $0.256 \pm 0.021^{\dagger * \#}$ & $<0.001$ & $-28.21 \pm 8.43^{\dagger}$ & $-36.73 \pm 5.95^{\dagger}$ \\
OVX + CMG & $0.413 \pm 0.019$ & $0.379 \pm 0.021^{\dagger \neq *}$ & $0.323 \pm 0.017^{\dagger \neq * \#}$ & $<0.001$ & $-8.13 \pm 4.94^{\dagger \neq}$ & $-21.52 \pm 3.99^{\dagger \neq}$ \\
$P$ value between groups & 0.705 & $<0.001$ & $<0.001$ & $<0.0005$ & $<0.0005$ \\
\hline
\end{tabular}

Comparison of absolutes values $\left(\mathrm{g} / \mathrm{cm}^{2}\right)$ and mean percentage changes of bone mineral density of the proximal tibia among groups measured at baseline (pre-ovariectomy), and at 3 and 6 months post-ovariectomy.

Values are mean \pm SD.

Significant differences at ${ }^{*} P<0.001$ vs. baseline, ${ }^{\#} P<0.001$ vs. 3 months, ${ }^{\dagger} P<0.001$ vs. control, and ${ }^{\ddagger} P<0.001$ vs. 0 VX.

OVX, ovariectomy; OVX + CMG, ovariectomy + Chios Mastic Gum.

was significantly higher than that of the OVX group at both 3 and 6 months (both $P<0.001$ ).

6 th lumbar vertebra: The results of the 6th lumbar vertebra are presented in Table 3. At baseline, there were no significant differences in BMD values between treatment groups. The BMD of the control group did not significantly differ during the 6 month treatment period $(P=0.238)$, whereas the BMD of the OVX group significantly decreased at 3 months ( $P=0.000$ vs. baseline) but did not significant differ between 3 and 6 months post treatment $(P=0.681)$. Similarly, the BMD of the OVX+CMG group significantly decreased at 3 months $(P=0.002$ vs. baseline) but stabilized between 3 months and 6 months $(P=$ $1.000)$. At 3 months, the $\mathrm{BMD}$ of the $\mathrm{OVX}+\mathrm{CMG}$ group was significantly higher than the BMD of the OVX group $(P=0.001)$, while there was no significant difference with the control group $(P=1.000)$. At 6 months, the BMD of the OVX+CMG group was significantly higher than the BMD of the OVX group $(P=0.010)$, with no significant difference versus the control group $(P=0.599)$.

\section{BMD percentage changes}

Total tibia: BMD percentage changes from baseline of the total tibia was evaluated for each group (Table 1). At 3 months of treatment, there was a significant difference between the control group $(4.41 \pm 4.15 \%)$, OVX $(-12.71$ $\pm 5.08 \%, P<0.001)$, or OVX+CMG $(-7.95 \pm 6.82 \%, P<$ $0.001)$ groups. At 6 months, the percentage change of the

Table 3. Comparison of the 6th lumbar vertebra between groups during the observation period of 6 months

\begin{tabular}{lcccccc}
\hline \multicolumn{1}{c}{ Group } & Baseline & 3 Months & 6 Months & $\begin{array}{c}P \text { value } \\
\text { within } \\
\text { group }\end{array}$ & $\begin{array}{c}\% \text { Change } \\
\text { baseline to } \\
3 \text { months }\end{array}$ & $\begin{array}{c}\% \text { Change } \\
\text { baseline to } \\
6 \text { months }\end{array}$ \\
\hline Control & $0.385 \pm 0.039$ & $0.358 \pm 0.042$ & $0.368 \pm 0.056$ & 0.238 & $-6.48 \pm 13.18$ & $-4.36 \pm 12.62$ \\
OVX & $0.382 \pm 0.047$ & $0.292 \pm 0.023^{\# \dagger \dagger}$ & $0.283 \pm 0.021^{\# \dagger}$ & $<0.001$ & $-22.75 \pm 9.22^{\dagger}$ & $-25.14 \pm 8.65^{\dagger \dagger}$ \\
OVX + CMG & $0.410 \pm 0.032$ & $0.352 \pm 0.029^{* \neq \dagger}$ & $0.334 \pm 0.047^{* \dagger}$ & $<0.001$ & $-13.79 \pm 7.32$ & $-15.81 \pm 10.35$ \\
$P$ value between groups & 0.284 & $<0.001$ & $<0.001$ & & 0.006 & 0.001 \\
\hline
\end{tabular}

Comparison of absolutes values $\left(\mathrm{g} / \mathrm{cm}^{2}\right)$ and mean percentage changes of bone mineral density of the 6th lumbar vertebra among groups measured at baseline (pre-ovariectomy), and at 3 and 6 months post-ovariectomy.

Values are mean $\pm S D$.

Significant differences at ${ }^{*} P<0.05$ and ${ }^{\#} P<0.005$ vs. baseline, ${ }^{\dagger} P<0.05$ and ${ }^{\dagger \dagger} P<0.005$ vs. control, and ${ }^{\ddagger} P<0.05$ and ${ }^{\ddagger \ddagger} P<0.005$ vs. 0 VX. OVX, ovariectomy; OVX + CMG, ovariectomy + Chios Mastic Gum. 
Table 4. Comparison of three-point-bending parameters of the right femur between the 3 groups

\begin{tabular}{lcccc}
\hline & Control & OVX & OVX+CMG & $P$ value \\
\hline von Mises stress (MPa) & $191.92 \pm 35.03$ & $179.90 \pm 34.75$ & $193.40 \pm 19.25$ & 0.597 \\
Bending stress (MPa) & $191.81 \pm 34.97$ & $179.68 \pm 34.78$ & $193.19 \pm 19.29$ & 0.594 \\
Torsional stress (MPa) & $3.13 \pm 2.37$ & $3.95 \pm 3.26$ & $4.78 \pm 2.14$ & 0.407 \\
Cross-sectional area $\left(\mathrm{mm}^{2}\right)$ & $5.02 \pm 0.45$ & $5.07 \pm 0.23$ & $5.09 \pm 0.45$ & 0.923 \\
Thickness $(\mathrm{mm})$ & $0.67 \pm 0.09$ & $0.58 \pm 0.05^{*}$ & $0.61 \pm 0.05$ & 0.016 \\
\hline
\end{tabular}

Values are mean \pm SD.

*Significant differences at $P=0.015$ vs. control.

OVX, ovariectomy; OVX + CMG, ovariectomy + Chios Mastic Gum.

control group $(5.01 \pm 8.03 \%)$ significantly differed from both the OVX $(-17.46 \pm 3.85 \%, P<0.001)$ and OVX+ CMG $(-10.63 \pm 6.75 \%, P<0.001)$ groups. There was no significant difference between the OVX+CMG and OVX at 3 nor 6 months ( $P=0.31$ and $P=0.19$, respectively).

Proximal tibia: BMD percentage changes from baseline of the proximal tibia was evaluated for each group (Table 2). After 3 months of treatment, there was a significant difference between the control $(2.05 \pm 5.98 \%)$, OVX $(-28.21$ $\pm 8.43 \%, P<0.001)$, and OVX+CMG $(-8.13 \pm 4.94 \%, P<$ 0.001 ) groups, and between the OVX and OVX + CMG groups $(P<0.001)$. The same results were evident at 6 months, whereby the percentage change of the control group $(2.89 \pm 5.56 \%)$ was significantly higher than the OVX $(-36.73 \pm 5.95, P<0.001)$ and OVX+CMG $(-21.52$ $\pm 3.99 \%, P<0.001)$ groups, and there were significant differences between the OVX and OVX+CMG groups $(P<0.001)$.

6th lumbar vertebra: BMD percentage changes of the 6th lumbar vertebra from baseline were evaluated for each group (Table 3). After 3 months of treatment, there was a significant difference between the control $(-6.48 \pm$ $13.18 \%)$ and OVX group $(-22.75 \pm 9.22 \%, P=0.005)$ but not the OVX+CMG group $(-13.79 \pm 7.32 \%, P=0.403)$. At 6 months, the percentage change of the control group $(-4.36 \pm 12.62 \%)$ was significantly higher than the OVX group $(-25.14 \pm 8.65 \%, P=0.005)$ but not the OVX+ CMG group $(-15.81 \pm 10.35 \%, P=0.083)$.

\section{Biomechanical testing}

Biomechanical parameters evaluated from the $3 \mathrm{pb}$ on the right femur included von Mises stress, bending stress, torsional stress, cross-sectional area, and thickness (Table 4). Statistical analysis showed no significant differences between the von Mises stress, bending stress, torsional stress, and cross-sectional area of the 3 groups. However, the thickness of the control group was significantly higher than the OVX group, but did not statistically differ from that of the OVX+CMG group.

\section{Cytologic evaluation of vaginal smears}

In the control group, vaginal cytology revealed regular, non-synchronized cycling, with expected stages of one-
Table 5. Comparison of the percentage of cornified cells between the 3 groups

\begin{tabular}{lcc}
\hline & $\begin{array}{c}\text { Cornified index } \\
<50 \%\end{array}$ & $\begin{array}{c}\text { Cornified index } \\
\geq 50 \%\end{array}$ \\
\hline Control & $0 / 10$ & $10 / 10$ \\
OVX & $10 / 10$ & $0 / 10$ \\
OVX+CMG & $10 / 10$ & $0 / 10$ \\
\hline
\end{tabular}

OVX, ovariectomy; OVX + CMG, ovariectomy + Chios Mastic Gum.

day proestrus, one-day estrous, one-day metestrus, and diestrous with a duration of 2 days. Normal estrogenic status was noted in the control group as a high percentage of cornified cells $(\geq 50 \%)$, which was absent in the $\mathrm{OVX}+\mathrm{CMG}$ and OVX groups. Administration of CMG did not affect the cornification index of the OVX+CMG group compared to the OVX group, as assessed by vaginal cytology (Table 5).

\section{BW}

All rats increased their BW during the study (Table 6). At baseline, animals in all groups were of a similar BW. At the end of the study, BW of the OVX+CMG and control groups did not significantly differ, whereas BW of the OVX group was significantly higher than that of the control group $(P<0.05)$. Similar differences were observed regarding intraperitoneal fat weight/BW (\%) of the 3 groups (Table 7).

\section{Uterine weight}

Mean uterine weight was significantly higher in the control group than the OVX and OVX+CMG groups. Differences in mean uterine weight between the OVX and OVX $+\mathrm{CMG}$ groups did not significantly differ (Table 7).

\section{Liver weight}

During the euthanasia and specimen collection, no morphological changes were observed in the livers of rats in any group. The mean liver weight of the OVX+CMG group was significantly higher than that of the OVX group, but did not significant differ from the control group (Table 7). 
Table 6. Comparison of body weight results between the 3 groups during the 6-month experimental period

\begin{tabular}{lccccccc}
\hline \multicolumn{1}{c}{ Group } & Baseline & Week 4 & Week 8 & Week 12 & Week 16 & Week 20 & Week 24 \\
\hline Control & $292.50 \pm 21.36$ & $293.60 \pm 23.56$ & $304.00 \pm 28.59$ & $317.00 \pm 36.30$ & $319.00 \pm 29.94$ & $323.70 \pm 34.27$ & $352.90 \pm 28.50$ \\
OVX & $297.70 \pm 30.19$ & $335.00 \pm 38.71^{* \#}$ & $335.40 \pm 39.67^{* \#}$ & $358.40 \pm 45.32^{*}$ & $374.20 \pm 51.49^{*}$ & $377.60 \pm 55.04^{*}$ & $399.00 \pm 61.68^{*}$ \\
OVX+CMG & $278.33 \pm 15.62$ & $287.33 \pm 19.21$ & $285.22 \pm 13.87$ & $325.67 \pm 18.23$ & $343.22 \pm 25.39$ & $346.78 \pm 26.27$ & $362.78 \pm 30.83$ \\
$P$ value & 0.136 & 0.002 & 0.004 & 0.038 & 0.011 & 0.023 & 0.062 \\
\hline
\end{tabular}

Values are mean \pm SD.

Significant differences at ${ }^{*} P<0.05$ vs. control and ${ }^{\#} P<0.05$ vs. $O V X+C M G$.

OVX, ovariectomy; OVX + CMG, ovariectomy + Chios Mastic Gum.

Table 7. Comparison of mean organ weight or mean organ weight/BW of the 3 groups

\begin{tabular}{lcccc}
\hline & Control & OVX & OVX+CMG & $P$ value \\
\hline Left kidney & $1.279 \pm 0.153$ & $1.057 \pm 0.080^{* *}$ & $0.998 \pm 0.090^{* *}$ & $<0.005$ \\
Uterus & $0.573 \pm 0.107$ & $0.197 \pm 0.054^{* *}$ & $0.152 \pm 0.028^{* *}$ & $<0.005$ \\
Liver & $10.907 \pm 1.472$ & $9.168 \pm 1.231^{*}$ & $11.890 \pm 1.441^{\dagger}$ & 0.001 \\
Intraperitoneal fat/BW (\%) & $6.542 \pm 0.646$ & $9.281 \pm 2.666^{* *}$ & $7.730 \pm 1.285$ & 0.007 \\
Gastrocnemius/BW (\%) & $0.506 \pm 0.031$ & $0.414 \pm 0.072^{* *}$ & $0.466 \pm 0.056$ & 0.006 \\
\hline
\end{tabular}

Values are mean \pm SD.

Significant differences at ${ }^{*} P<0.05$ and ${ }^{* *} P<0.005$ vs. control, and ${ }^{\dagger} P<0.05$ vs. OVX.

$\mathrm{BW}$, body weight. OVX, ovariectomy; OVX + CMG, ovariectomy + Chios Mastic Gum.

\section{Left kidney}

The mean left kidney weight of the control group was significantly higher than that of the OVX and OVX+ CMG groups. Differences in mean left kidney weights of the OVX and OVX+CMG groups did not significantly differ (Table 7).

\section{Intraperitoneal fat weight/BW (\%)}

The mean intraperitoneal fat weight/BW (\%) of the OVX group was significantly higher than that of the control group. No significant differences in the mean intraperitoneal fat weight/BW (\%) were observed between the OVX and OVX+CMG groups, or between the control and OVX+CMG groups (Table 7).

\section{Gastrocnemius muscle weight/BW (\%)}

The mean gastrocnemius weight/BW (\%) of the control group was significantly higher than the OVX group. No significant differences in the mean gastrocnemius weight/ BW (\%) were observed between the OVX and OVX+ CMG groups, or between the control and OVX+CMG groups (Table 7).

\section{DISCUSSION}

The present study investigated the effect of CMG administration on mature ovariectomized rats. CMG constitutes an exceptional Greek product, produced exclusively in the southern part of the island of Chios. CMG is the aromatic resin produced by the evergreen shrub Pistacia lentiscus var. Chia (Anacardiaceae), obtained from shallow inci- sions made on the bark and trunk of the shrub. A plethora of references report use of CMG in traditional medicine, dating from the ancient times of Galen, Theophrastus, and Dioscorides. Today, CMG resin has been rediscovered as both a traditional remedy and as a phytotherapeutic product with numerous biological properties (Ierapetritis, 2010; Pachi et al., 2020).

From a chemical perspective, CMG constituents are categorized into four main groups. Specifically, the constituents may be divided into the acidic fraction (triterpenic acids; $\sim 38 \%$ of total CMG), neutral fraction (other triterpenes; $\sim 27 \%$ of total $\mathrm{CMG}$ ), poly- $\beta$-myrcene (natural polymer; $25 \sim 30 \%$ of $\mathrm{CMG}$ ), and other minor compounds together with CMG essential oil (almost $15 \mathrm{~mL}$ / kg) (Pachi et al., 2020; Pachi et al., 2021). Of importance, terpenes comprise approximately $70 \%$ of CMG constituents that make this resin unique vs. other phytotherapeutics. Another herbal medicine, C. rhizome (Actaea racemose or Cimicifuga racemosa-black Cohosh), is characterized by the presence of terpenes, particularly triterpenes, and its use in the treatment of menopause symptoms is highly acknowledged (Geller and Studee, 2006; Office of Dietary Supplements, 2019). Based on the above, we evaluated the effects of CMG on boss loss.

The mature ovariectomized rat model has been extensively used to demonstrate bone-protective effects of new therapeutic agents, including many plant extracts. In the present study, after ovariectomy, the BMD of the total tibia, proximal tibia and 6th lumbar vertebra was significantly decreased.

After 6 months of CMG administration, although the $\mathrm{BMD}$ of the total tibia of the OVX+CMG group was de- 
creased significantly vs. baseline $(P<0.001)$, it was significantly higher than the OVX group. Similarly, treatment of OVX rats with Onobrychis ebenoides plant extracts significantly increased the BMD in rats compared with untreated OVX rats (Dontas et al., 2006). Furthermore, blueberries prevented bone loss in ovariectomized rats, as shown increases in BMD of total tibia vs. OVX rats (Devareddy et al., 2008). In addition, Glycyrrhiza glabra root extracts show a protective effect on BMD of total tibia following administration for 6 months (Galanis et al., 2019). However, 6 months administration of Amphimas pterocarpoides does not elicit a protective effect on the total tibia BMD (Patsaki et al., 2016).

Similar results were observed on the proximal tibia after administration of CMG for 6 months. Although the $\mathrm{BMD}$ of the OVX+CMG group was significantly decreased after 6 months, it was significantly higher than the OVX group. Similarly, administration of O. ebenoides significantly increased BMD of proximal tibia of OVX rats vs. untreated OVX rats (Dontas et al., 2006). Furthermore, 6 months of treatment with G. glabra extract (Galanis et al., 2019) and A. pterocarpoides (Patsaki et al., 2016) also showed protective effects on BMD of proximal tibia.

At both 3 and 6 months, absolute BMD of the 6th lumbar vertebra of the OVX+CMG group was significantly higher than the BMD of the OVX group, with no significant difference vs. the control group. In a previous study that investigated the effect of different doses of simvastatin on BMD of the fourth lumbar vertebrae of OVX rats, simvastatin did not had elicited higher BMD vs untreated OVX rats, although these results were not considered significant (Long et al., 2016).

No difference in von Mises, bending or torsional stresses were observed between groups during the 6 months study period. In a previous study, ovariectomized rats showed mid-diaphyseal endosteum with increased bone resorption, which enlarged the size of the medullary cavity (Pan et al., 1995). Concurrently, ovariectomy stimulates periosteal bone growth in the cortical bone of the diaphysis of the long bones (Miller et al., 1991). Because of these conjunctional events, resistance/strength of the femur changes at a slow rate (Kalu, 1991). According to Jee and Yao (2001), changes in the biomechanical strength of bones can be observed from nine months post ovariectomy. Similarly to our study, Galanis et al. (2019) observed a highly significant increase in BMD following administration of G. glabra in ovariectomized rats, with no significant effect on bone strength. However, the fracture stress of OVX rats treated with A. pterocarpoides was significantly greater than OVX rats (Patsaki et al., 2016), and OVX rats treated with Sideritis euboea had significantly higher maximal load before fracture compared to OVX rats (Dontas et al., 2011). In another study, the thickness of the femoral shaft decreased after 3 months of the ovariectomy (Danielsen et al., 1993). In our study, the thickness of the right femur of the OVX+CMG group had an intermediate value, which was lower than the control group but higher than the OVX group, indicating a protective effect of CMG.

In the present study, the uterine weight of OVX and OVX+CMG rats did not significantly differ, but were significantly lower than the control group. Of our four previous studies of rats treated for 6 months (Dontas et al., 2006; Dontas et al., 2011; Patsaki et al., 2016; Galanis et al., 2019) and two other studies of rats treated for 3 months (Danielsen et al., 1993; Devareddy et al., 2008), five showed uterine weights of all OVX rats were similar to treated groups but significantly lower than control groups, indicating a lack of effect of these plant extracts on the uterus. However, in the study by Galanis et al. (2019) the uterine weight of OVX rats treated with G. glabra was between those of the OVX and control group, and did not statistically differ from either.

The lack of effect of CMG on uterine weight was consistent with the cytological evaluation of the vaginal smears, which showed that CMG does not impact the cornification index of the vagina. The cornification index is accepted as a precise indicator of the impact of estrogen in the vaginal epithelium of women (Wood et al., 1961). Furthermore, exposure to estrogenic compounds have been reported to result in a persistent vaginal cornification indexes in laboratory rodents (Goldman et al., 2007). In our study, both the OVX and OVX+CMG groups had cornification indexes lower than $50 \%$, which suggests no estrogenic action of CMG.

In the present study, all 3 groups had an increase in mean BW. The OVX+CMG had an intermediate value that did not significantly differ from the other two groups, while the OVX group significantly differed from the control group. In addition, the mean intraperitoneal fat/BW (\%) of the OVX+CMG group did not significantly differ from either the control or OVX groups. This observation may be of high importance, because in human abdominal obesity has been positively associated with a higher risk of hip fractures (Sadeghi et al., 2017). The mean gastrocnemius weight/BW (\%) of the OVX+CMG group did not differ from the control group but was higher than the OVX group, although the difference was not considered significant. However, the mean gastrocnemius weight/ BW (\%) was significantly lower in the OVX group than the control group. These results suggest a strong beneficial impact of CMG on the muscular system. The strength of muscles in humans is inversely associated with risk of falls and fractures (Wickham et al., 1989). Consequently, this beneficial effect of CMG on muscles may have a favorable impact on other concurrent risk factors for fractures related to post-menopausal osteoporosis. Adminis- 
tration of other plant extracts failed to protect intervention groups from increased BW relative to OVX rats (Dontas et al., 2006; Devareddy et al., 2008; Patsaki et al., 2016).

Increased nuclear factor-kappa B (NF- $\kappa \mathrm{B})$ activity has been identified in several in vivo studies as a key factor that mediates muscle atrophy (Thoma and Lightfoot, 2018). In our study, the mean gastrocnemius weight/BW (\%) of the OVX+CMG group did not significantly differ from the control group, which corresponds with research by $\mathrm{He}$ et al. (2006) that showed that CMG blocked the NF- $\mathrm{kB}$ activity and pathway.

Kang et al. (2007) investigated the potential toxicological impact of different doses of CMG administered in the diet of young rats on liver function. The study reported that CMG had an impact on absolute liver weight values, although no morphological alterations were observed during the necropsy (Kang et al., 2007). Through biochemical analysis of the liver parameters, the changes observed in the liver were not considered related to any adverse effect of CMG (Kang et al., 2007). In our study, the concentration of CMG was intermediate between the medium and higher concentrations of the previous study, and our results are comparable (liver weight and absence of gross morphological lesions on necropsy) to the previous study. Therefore, we hypothesize that the differences we observed in liver weight was not associated with any adverse effects.

The differences in mean kidney weight between OVX + CMG and OVX groups were not significant, whereas both groups significantly differed from the control. In a study conducted by Das et al. (2004), a statistical difference was observed between OVX and control groups, while OVX rats administered black tea extracts did not statistically differ from the control group. However, a direct comparison to our study is not straightforward due to the shorter observation period (Das et al., 2004). Other studies have not able to document such differences between OVX and control groups (Patsaki et al., 2016; Vorland et al., 2019), and other plant extracts administered in comparable post-menopausal studies had no effect on kidney weight (Patsaki et al., 2016). However, in a study that evaluated the toxicological impact of CMG on kidneys, differences observed in biochemical analysis were not considered to reflect a toxicological impact on renal function (Kang et al., 2007). Further investigation is needed to elucidate the mechanism (if any) by which the differences observed in kidney weight occurred.

CMG has been used since antiquity and although many of its healing and beneficial properties have been studied, its effect on bone metabolism has not been previously investigated. Since 2000, several studies have investigated the anti-microbial, antioxidant, anti-inflammatory, and anti-cancer activities of CMG (Andrikopoulos et al., 2003;
He et al., 2006; Triantafyllou et al., 2011; Georgiadis et al., 2015). Plant products are comprised from several substances, each of which may trigger different pathways. Bone metabolism is usually affected by anti-inflammatory action, phytoestrogens or via the NF- $\mathrm{\kappa B}$ pathway (Suzuki et al., 2020). In a study on androgen-responsive prostate

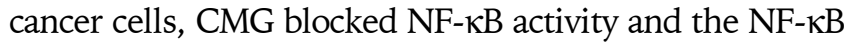
pathway (He et al., 2006). In addition, a study on experimental colitis showed that CMG reduces inflammation via NF- $\kappa B$ regulation (Papalois et al., 2012). Activation of $\mathrm{NF}-\kappa \mathrm{B}$ in most cells is triggered by a wide range of stimuli, such as cytokines, immune modulators, and other stresses (Jimi et al., 2019). Many members of the NF- $\mathrm{kB}$ family are implicated in development and activity of osteoclasts and early inflammatory responses (Boyce, 2013; Jimi et al., 2019). Furthermore, CMG exerts a strong and well-documented antioxidant activity (Andrikopoulos et al., 2003; Georgiadis et al., 2015). Consequently, we hypothesize that the beneficial effect of CMG on bone metabolism and muscle mass may be due to its antioxidant and anti-inflammatory action.

A significant limitation of this study is the duration of the observation period for biomechanical parameters. According to the literature, changes in biomechanical parameters may need observation periods as long as nine months (Kalu, 1991). Although the mean life span of a female rat is approximately $813 \pm 214$ days (Fukuda and Iida, 2003), clinical or subclinical age-related degenerative diseases (tumors, respiratory infections, renal, or cardiac disorders) (Hirokawa, 1975; Fukuda and Iida, 2003) that often appear after one year of age may have altered or implicated the results. Furthermore, collection of blood and urine samples for estimation of bone biomarkers would have provided additional information regarding the first period of rapid bone loss following ovariectomy. However, blood and urine collection would subject the rats to significant stress (Hoffman et al., 2018; Sahin et al., 2019), which could interfere with the results. In addition, in animals with established osteopenia, interpretation of bone biomarkers may not provide valid information of their turnover (Turner et al., 2001). Another limitation of this study was the lack of bone histomorphometry measurements. Static and dynamic parameters of bone histomorphometry at euthanasia would have provided additional information regarding the CMG effects on bone. However, due to limited technical resources, this was not feasible.

CMG administered to an OVX rat model of postmenopausal osteoporosis showed a protective effect on bone mineral density of both the total and proximal tibia and the 6th lumbar vertebra. BW, intraperitoneal fat weight, and gastrocnemius weight results indicate that administration of CMG may significantly reduce risk factors associated with falls and fractures in humans. Uterine weight 
and cytological evaluation of vaginal smears demonstrated that CMG did not have an estrogenic effect. This protective effect on ovariectomy-induced bone loss, accompanied by lack of an undesirable effect on the uterus, indicates CMG consumption has potential in addition to its known beneficial properties.

\section{ACKNOWLEDGEMENTS}

The authors wish to thank Mrs. C. Kapsi and Mrs. G. Mastrogianni for their assistance in performing the ovariectomies and the autopsies, Mr. G. Kiniklis for assisting in the DEXA scans, and the Chios Mastiha Growers Association for providing the Chios Mastic Gum powder. Vasiliki K Pachi is co-financed by Greece and the European Union (European Social Fund-ESF) through the Operational Programme 《Human Resources Development, Education and Life long Learning $》$ in the context of the project "Strengthening Human Resources Research Potential via Doctorate Research" implemented by the State Scholarships Foundation (ILY).

\section{AUTHOR DISCLOSURE STATEMENT}

The authors declare no conflict of interest.

\section{REFERENCES}

Almeida M, Han L, Martin-Millan M, Plotkin LI, Stewart SA, Roberson PK, et al. Skeletal involution by age-associated oxidative stress and its acceleration by loss of sex steroids. J Biol Chem. 2007. 282:27285-27297.

Andreadou I, Mitakou S, Paraschos S, Efentakis P, Magiatis P, Kaklamanis L, et al. "Pistacia lentiscus L." reduces the infarct size in normal fed anesthetized rabbits and possess antiatheromatic and hypolipidemic activity in cholesterol fed rabbits. Phytomedicine. 2016. 23:1220-1226.

Andrikopoulos NK, Kaliora AC, Assimopoulou AN, Papapeorgiou VP. Biological activity of some naturally occurring resins, gums and pigments against in vitro LDL oxidation. Phytother Res. 2003. 17:501-507.

Beral V; Million Women Study Collaborators, Bull D, Green J, Reeves G. Ovarian cancer and hormone replacement therapy in the Million Women Study. Lancet. 2007. 369:1703-1710.

Boyce BF. Advances in the regulation of osteoclasts and osteoclast functions. J Dent Res. 2013. 92:860-867.

Danielsen CC, Mosekilde L, Svenstrup B. Cortical bone mass, composition, and mechanical properties in female rats in relation to age, long-term ovariectomy, and estrogen substitution. Calcif Tissue Int. 1993. 52:26-33.

Das AS, Mukherjee M, Mitra C. Evidence for a prospective antiosteoporosis effect of black tea (Camellia Sinensis) extract in a bilaterally ovariectomized rat model. Asia Pac J Clin Nutr. 2004. 13:210-216.

Devareddy L, Hooshmand S, Collins JK, Lucas EA, Chai SC, Arjmandi $\mathrm{BH}$. Blueberry prevents bone loss in ovariectomized rat model of postmenopausal osteoporosis. J Nutr Biochem.
2008. 19:694-699.

Dontas I, Halabalaki M, Moutsatsou P, Mitakou S, Papoutsi Z, Khaldi L, et al. Protective effect of plant extract from Onobrychis ebenoides on ovariectomy-induced bone loss in rats. Maturitas. 2006. 53:234-242.

Dontas IA, Lelovas PP, Kourkoulis SK, Aligiannis N, Paliogianni A, Mitakou S, et al. Protective effect of Sideritis euboea extract on bone mineral density and strength of ovariectomized rats. Menopause. 2011. 18:915-922.

Erben RG. Trabecular and endocortical bone surfaces in the rat: modeling or remodeling?. Anat Rec. 1996. 246:39-46.

European Medicines Agency. Assessment report on Cimicifuga racemosa (L.) Nutt., rhizoma. 2017 [cited 2017 Jul 18]. Available from: https://www.ema.europa.eu/en/documents/herbalreport/draft-assessment-report-cimicifuga-racemosa-l-nuttrhizome-revision-1_en.pdf

European Medicines Agency. Assessment report on Pistacia lentiscus L., resina (mastic). 2015 [cited 2016 Feb 2]. Available from: https://www.ema.europa.eu/en/documents/herbal-report/fi nal-assessment-report-pistacia-lentiscus-l-resin-mastic_en.p df

Fukuda S, Iida $\mathrm{H}$. Life span and spontaneous tumors incidence of the Wistar Mishima (WM/MsNrs) rat. Exp Anim. 2003. 52: 173-178.

Galanis D, Soultanis K, Lelovas P, Zervas A, Papadopoulos P, Galanos A, et al. Protective effect of Glycyrrhiza glabra roots extract on bone mineral density of ovariectomized rats. Biomedicine. 2019. 9:8. https://doi.org/10.1051/bmdcn/2019090208

Geller SE, Studee L. Contemporary alternatives to plant estrogens for menopause. Maturitas. 2006. 55:S3-S13.

Georgiadis I, Karatzas T, Korou LM, Katsilambros N, Perrea D. Beneficial health effects of Chios Gum Mastic and peroxisome proliferator-activated receptors: indications of common mechanisms. J Med Food. 2015. 18:1-10.

Giaginis C, Theocharis S. Current evidence on the anticancer potential of Chios mastic gum. Nutr Cancer. 2011. 63:1174-1184.

Goldman JM, Murr AS, Cooper RL. The rodent estrous cycle: characterization of vaginal cytology and its utility in toxicological studies. Birth Defects Res B Dev Reprod Toxicol. 2007. 80:8497.

He ML, Yuan HQ, Jiang AL, Gong AY, Chen WW, Zhang PJ, et al. Gum mastic inhibits the expression and function of the androgen receptor in prostate cancer cells. Cancer. 2006. 106:25472555.

Hirokawa K. Characterization of age-associated kidney disease in Wistar rats. Mech Ageing Dev. 1975. 4:301-316.

Hoffman JF, Fan AX, Neuendorf EH, Vergara VB, Kalinich JF. Hydrophobic sand versus metabolic cages: a comparison of urine collection methods for rats (Rattus norvegicus). J Am Assoc Lab Anim Sci. 2018. 57:51-57.

Ierapetritis D. The geography of the Chios Mastic trade from the 17th through to the 19th century. Ethnobot Res Appl. 2010. 8:153-167.

Jee WS, Yao W. Overview: animal models of osteopenia and osteoporosis. J Musculoskelet Neuronal Interact. 2001. 1:193-207.

Jimi E, Fei H, Nakatomi C. NF- $\kappa B$ signaling regulates physiological and pathological chondrogenesis. Int J Mol Sci. 2019. 20:6275. https://doi.org/10.3390/ijms20246275

Kalu DN. The ovariectomized rat model of postmenopausal bone loss. Bone Miner. 1991. 15:175-191.

Kang JS, Wanibuchi H, Salim EI, Kinoshita A, Fukushima S. Evaluation of the toxicity of mastic gum with 13 weeks dietary administration to F344 rats. Food Chem Toxicol. 2007. 45:494501.

Lacativa PGS, de Farias MLF. Osteoporosis and inflammation. Arq Bras Endocrinol Metabol. 2010. 54:123-132.

Lelovas PP, Xanthos TT, Thoma SE, Lyritis GP, Dontas IA. The 
laboratory rat as an animal model for osteoporosis research. Comp Med. 2008. 58:424-430.

Lemonakis N, Magiatis P, Kostomitsopoulos N, Skaltsounis AL, Tamvakopoulos C. Oral administration of Chios mastic gum or extracts in mice: quantification of triterpenic acids by liquid chromatography-tandem mass spectrometry. Planta Med. 2011. 77:1916-1923.

Li JX, Liu J, He CC, Yu ZY, Du Y, Kadota S, et al. Triterpenoids from Cimicifugae rhizoma, a novel class of inhibitors on bone resorption and ovariectomy-induced bone loss. Maturitas. 2007. 58:59-69.

Li JX, Yu ZY. Cimicifugae rhizoma: from origins, bioactive constituents to clinical outcomes. Curr Med Chem. 2006. 13:29272951.

Long T, Tang T, Hao Y, Zhu Z, Shao L, Liu M. Effect of simvastatin on osteogenesis of the lumbar vertebrae in ovariectomized rats. Exp Ther Med. 2016. 12:3951-3957.

Maggio D, Barabani M, Pierandrei M, Polidori MC, Catani M, Mecocci P, et al. Marked decrease in plasma antioxidants in aged osteoporotic women: results of a cross-sectional study. J Clin Endocrinol Metab. 2003. 88:1523-1527.

Miller SC, Bowman BM, Miller MA, Bagi CM. Calcium absorption and osseous organ-, tissue-, and envelope-specific changes following ovariectomy in rats. Bone. 1991. 12:439-446.

Nair AB, Jacob S. A simple practice guide for dose conversion between animals and human. J Basic Clin Pharm. 2016. 7:27-31.

Office of Dietary Supplements. Black cohosh: fact sheet for health professionals. 2019 [cited 2021 Jan 20]. Office of Dietary Supplements, National Institutes of Health, Bethesda, MD, USA. Available from: https://ods.od.nih.gov/factsheets/BlackCohosh-HealthProfessional/

Pachi VK, Mikropoulou EV, Dimou S, Dionysopoulou M, Argyropoulou A, Diallinas G, et al. Chemical profiling of Pistacia lentiscus var. Chia resin and essential oil: ageing markers and antimicrobial activity. Processes. 2021. 9:418. https://doi.org/10. 3390/pr9030418

Pachi VK, Mikropoulou EV, Gkiouvetidis P, Siafakas K, Argyropoulou A, Angelis A, et al. Traditional uses, phytochemistry and pharmacology of Chios mastic gum (Pistacia lentiscus var. Chia, Anacardiaceae): a review. J Ethnopharmacol. 2020. 254: 112485. https://doi.org/10.1016/j.jep.2019.112485

Pan Z, Jee WS, Ma YF, McOsker JE, Li XJ. Intermittent treatments of prostaglandin E2 plus risedronate and prostaglandin E2 alone are equally anabolic on tibial shaft of ovariectomized rats. Bone. 1995. 17:291S-296S.

Papalois A, Gioxari A, Kaliora AC, Lymperopoulou A, Agrogiannis G, Papada E, et al. Chios mastic fractions in experimental colitis: implication of the nuclear factor $\kappa B$ pathway in cultured HT29 cells. J Med Food. 2012. 15:974-983.

Paraschos S, Magiatis P, Mitakou S, Petraki K, Kalliaropoulos A, Maragkoudakis $\mathrm{P}$, et al. In vitro and in vivo activities of Chios mastic gum extracts and constituents against Helicobacter pylori. Antimicrob Agents Chemother. 2007. 51:551-559.

Patsaki A, Tchoumtchoua J, Passali C, Lelovas P, Kourkoulis S,
Papaioannou N, et al. The protective effect of Amphimas pterocarpoides plant extract on bone mineral density and strength in estrogen deficient ovariectomized Wistar rats. Med Aromat Plants. 2016. 5:269. https://doi.org/10.4172/2167-0412. 1000269

Rachner TD, Khosla S, Hofbauer LC. Osteoporosis: now and the future. Lancet. 2011. 377:1276-1287.

Rossouw JE, Anderson GL, Prentice RL, LaCroix AZ, Kooperberg C, Stefanick ML, et al. Risks and benefits of estrogen plus progestin in healthy postmenopausal women: principal results from the Women's Health Initiative randomized controlled trial. JAMA. 2002. 288:321-333.

Sadeghi O, Saneei P, Nasiri M, Larijani B, Esmaillzadeh A. Abdominal obesity and risk of hip fracture: a systematic review and meta-analysis of prospective studies. Adv Nutr. 2017. 8:728738.

Sahin Z, Solak H, Koc A, Ozen Koca R, Ozkurkculer A, Cakan P, et al. Long-term metabolic cage housing increases anxiety/depression-related behaviours in adult male rats. Arch Physiol Biochem. 2019. 125:122-127.

Sontakke AN, Tare RS. A duality in the roles of reactive oxygen species with respect to bone metabolism. Clin Chim Acta. 2002. 318:145-148.

Suzuki A, Sugiyama G, Ohyama Y, Kumamaru W, Yamada T, Mori Y. Regulation of NF- $\kappa B$ signalling through the PR55 $\beta$-RelA interaction in osteoblasts. In Vivo. 2020. 34:601-608.

Thoma A, Lightfoot AP. NF- $\kappa B$ and inflammatory cytokine signalling: role in skeletal muscle atrophy. Adv Exp Med Biol. 2018. 1088:267-279.

Triantafyllou A, Bikineyeva A, Dikalova A, Nazarewicz R, Lerakis S, Dikalov S. Anti-inflammatory activity of Chios mastic gum is associated with inhibition of TNF-alpha induced oxidative stress. Nutr J. 2011. 10:64. https://doi.org/10.1186/14752891-10-64

Turner CH, Burr DB. Basic biomechanical measurements of bone: a tutorial. Bone. 1993. 14:595-608.

Turner RT, Maran A, Lotinun S, Hefferan T, Evans GL, Zhang M, et al. Animal models for osteoporosis. Rev Endocr Metab Disord. 2001. 2:117-127.

Vorland CJ, Lachcik PJ, Swallow EA, Metzger CE, Allen MR, Chen $\mathrm{NX}$, et al. Effect of ovariectomy on the progression of chronic kidney disease-mineral bone disorder (CKD-MBD) in female Cy/+ rats. Sci Rep. 2019. 9:7936. https://doi.org/10.1038/ s41598-019-44415-9

Wickham C, Cooper C, Margetts BM, Barker DJ. Muscle strength, activity, housing and the risk of falls in elderly people. Age Ageing. 1989. 18:47-51.

Wood C, Osmond-Clarke F, Murray M. Vaginal cytology in pregnancy. J Obstet Gynaecol Br Emp. 1961. 68:778-783.

Xynos N, Termentzi A, Fokialakis N, Skaltsounis LA, Aligiannis N. Supercritical $\mathrm{CO}_{2}$ extraction of mastic gum and chemical characterization of bioactive fractions using LC-HRMS/MS and GC-MS. J Supercrit Fluids. 2018. 133:349-356. 